\title{
The Author Reply to Comment on "A Simple Method for International Standardization of Photographic Documentation for Aesthetic Plastic Surgery"
}

\author{
Seung Chul Rhee ${ }^{1}$ (1)
}

Received: 13 December 2017/ Accepted: 24 December 2017/Published online: 4 January 2018

(C) Springer Science+Business Media, LLC, part of Springer Nature and International Society of Aesthetic Plastic Surgery 2017

Level of Evidence $V$ This journal requires that authors assign a level of evidence to each article. For a full description of these Evidence-Based Medicine ratings, please refer to the Table of Contents or the online Instructions to Authors www.springer.com/00266.

I would like to sincerely thank you for sharing several valuable and noteworthy suggestions regarding the ideas presented in my article about photogrammetric standardization of facial photographs. For the past 13 years, I have conducted a substantive literature review on the standardization for diagnostic facial photographs and performed many experiments by reproducing, modifying and expanding upon these methods and techniques myself during that time. The practical and simple method approach set forth in my article was developed through much trial and error. However, there are limitations, and it is still too early to adopt as an international standard without further peer review. I am looking forward to more colleagues to share, contribute and further the development of guidelines to standardize digital facial photographic documentation for plastic surgeons worldwide. All three of the suggestions and points you have mentioned are correct and can alleviate limitations in my methodology. I tried to consider both the worst and most typical photographic conditions of small private practices and clinics, bearing in mind that doctors may want to further simplify diagnostic photographs with devices such as smartphone cameras in

Seung Chul Rhee

artprs@naver.com

1 Department of Plastic and Reconstructive Surgery, Ilsan Hospital, Dongguk University College of School of Medicine, 27 Dongguk-ro, Ilsandong-gu, Goyang City, Kyounggi-Do 410-773, Republic of Korea the future. I intentionally tried to take facial photographs without sophisticated lighting arrangements in a small clinic setting while using low-priced camera equipment. However, this methodology inherently produces a few issues and limitations as you have sensibly pointed out.

First, as you indicated, the oblique shadows can be seen from the sample photographs. This was the result of using the built-in flash attached to the camera under the lighting conditions of a small-quarters clinic as opposed to a photography studio. It is more appropriate to use a ring light or two studio-grade strobe lights as you suggested. The second point you recommended is to place the RGB and CMYK circles on both sides of the subject instead of on one side. This is a very good and constructive suggestion that I believe will further advance achieving international photographic standardization. Finally, you also recommended that the diameter of the camera lens should be $70 \mathrm{~mm}$ or more. You are correct, but for this study I used a low-priced and entry-level DSLR (Cannon EOS 650D). The trade-off between low cost equipment and quality are apparent with the blurring of the background that you have noticed. I believe that the method, necessity, and technical advancement for international standardization of facial photographs will be determined through our colleagues' interest and collective scholastic conscience. I would like to extend my sincere gratitude for your interest, suggestions and recommendations regarding my article to further advance this endeavor. I wish our international journal, "Aesthetic Plastic Surgery", would give absolute guidelines for standardizing digital photographic documentation. Thank you.

\section{Compliance with Ethical Standards}

Conflict of interest The authors declare that they have no conflict of interest. 